\title{
Influence of various application types on the performance of LTE mobile networks
}

\author{
Adel Agamy, Ahmed M. Mohamed \\ Electrical Engineering Department, Faculty of Engineering, Aswan University, Egypt
}

\begin{abstract}
Article Info
Article history:

Received Jan 16, 2020

Revised Apr 16, 2020

Accepted Apr 29, 2020

\section{Keywords:}

LTE

Queuing theory

Traffic modeling

Wireless network

ABSTRACT

Modern mobile internet networks are becoming heavier and denser. Also it is not regularly planned, and becoming more heterogeneous. The explosive growth in the usage of smartphones poses numerous challenges for LTE cellular networks design and implementation. The performance of LTE networks with bursty and self-similar traffic has become a major challenge. Accurate modeling of the data generated by each connected wireless device is important for properly investigating the performance of LTE networks. This paper presents a mathematical model for LTE networks using queuing theory considering the influence of various application types. Using sporadic source traffic feeding to the queue of the evolved nodeB and with the exponential service time assumption, we construct a queuing model to estimate the performance of LTE networks. We use the performance model presented in this paper to study the influence of various application categories on the performance of LTE cellular networks. Also we validate our model with simulation using NS3 simulator with different scenarios.
\end{abstract}

Copyright (c) 2020 Insitute of Advanced Engineeering and Science. All rights reserved.

\section{Corresponding Author:}

Adel Agamy,

Electrical Engineering Department, Faculty of Engineering,

Aswan University, Aswan, 81542, Egypt

Email: a.f.agamy@aswu.edu.eg

\section{INTRODUCTION}

The incredible growth in the number of wireless devices such as smart-phones, tablets and Internet of Thing (IoT) in addition to the fast development of media streaming applications, IPTV, telemedicine and Internet gaming have led to a significant challenge to the design and deployment of cellular technology. The mobile networks specifically the LTE networks are used only for accommodating voice and video calls traffic which are considered real time application. Also nowadays the mobile networks are used to transfer non-real time data (Email, ftp, ..etc). Each application type demands to maintain a certain level of quality (throughput, delay..etc) during his sojourn time through the LTE network [1, 2, 3, 4]. Investigating and analyzing the distribution of data generated by each device in LTE network should be the most important factor to estimate the quality requirements and capabilities of LTE networks. In this research we investigate and analyze (by analytical modeling and simulation) the influence of various application types on the LTE network performance. Also we validate our model through real network simulator NS3 with various scenarios. Our paper is organized as follows, previous studies related to the paper topic is presented in section II. The LTE network system model and its parameters are presented in section III. Section IV shows the LTE network performance estimation using analytical analysis with network performance metrics such as end to end packet delay and blockage probability. Section V shows the performance behavior of the LTE network using NS3 simulator with different operation scenarios. In section VII we conclude our work. 


\section{RELATED WORK}

Sajid et all in [5] developed a model using two different distributions. For Constant bit Rate (CBR) traffic (Ex. VOIP traffic) they used exponential distribution. To model Variable Bit Rate (VBR) traffic (video streaming) they used fractional brownie motion (FBM) traffic with heavy-tailed Weibull distribution for buffer occupancy [6]. The authors in [7] investigated the performance of cognitive radio links subject to recurrent failures and interruptions. They studied the performance with single and multiple channels. Using the queuing model $\mathrm{M} / \mathrm{M} / 1$, they considered the service interruption from primary users, also they used the pricing policy to charge each secondary user in the queue. Authors in [8] proposed a queueing model with four different priority queueing disciplines to apply dynamic optimization. They considered a dynamic priority queueing discipline to optimize a joint performance utility function on two classes of cognitive radio.

The performance of vehicular network communication using cellular LTE network using queueing models is presented in $[9,10,11,12,13]$. For example the author in [11] developed an analytical model describing the performance of periodic broadcast in Vehicular Ad-hoc Networks (VANET) in terms of packet collision probability and average packet delay using $\mathrm{M} / \mathrm{M} / \infty$ queuing model. Analytical models to evaluate the queue length behavior at the intersection points as a function of the percentage of vehicles are presented in [12]. The authors in [13] used deterministic arrival process and the queueing model D/M/1 for studying the performance of periodic broadcast in VANETs using metrics such as the packet collision probability and average packet delay. The authors in [14] used the queuing theory to develop a model for cellular LTE wireless networks. They assumed that the cellular LTE network is serving variable bit-rate calls. The authors in [15] evaluated the polling behavior on a MAC for cellular network analytically considering the packet delay, buffer overflow rates and energy consumption. Also in their work in [16] they introduced analytical models to characterize the delay for multicast transmission over a communication channel model. In [17], authors presented a mixed queueing network models of several mobility users at numerous access points to accurately predicting the number of network-level performance and user-level performance in a wireless network. In [18] Scott et all introduced a model for Vehicular Wireless Channel Communication, they modeled the LTE system channel with M/M/m Queueing model. They modeled the LTE wireless communication channel with $\mathrm{M} / \mathrm{M} / \mathrm{m}$ queueing model with infinite number of resources channel $\mathrm{m}$. Also they assumed that each vehicle generates traffic with Poisson process of exponential inter arrival time distribution. They used the First Come First Send (FCFS) queueing discipline to handle traffic in the queue. They evaluated the probability that channels being busy, also developed the expected waiting times and the expected number of channel switches. Fowler et all in [19] described the behavior of the LTE video call (ex. Skype video call) and video streaming traffic in heterogeneous real environment using gaussian mixture model. They derived a semi-Markov model with six states for video call and they derived a semi-Markov model with fifteen states to fit the statistics of composite LTE video measurements.

Najem et all in [20] used the Disjoint Queue Scheduler (DQS) for the LTE-A heterogeneous network deployment of a macro-cell and a variable number of picocells. They evaluated and compared the Quality of Service (QoS) of the user performance using the DQS based on different techniques. They evaluated the QoS using the average subscriber's metrics throughput, Packet Loss Rate (PLR), and average packet delay. They experimentally evaluated the performance of the DQS and its effect on the user quality of service. Naumov et all in [21] studied the performance of LTE cellular networks using queueing models with limited resources. They developed a mathematical model for resource sharing in LTE cellular network using multi-server queueing model. They assumed that users arrive in the system as independent Poisson flows. The service time for each arrival is modeled with exponential distribution. The authors considered a multi-server queuing model and they used semi-markov chains to derive the stationary probabilities for the LTE cellular network with single LTE networks serving users using video conference call. Polaganga et all in [22] explored the Self-Similarity property of LTE and LTE-advance cellular networks. They showed that the selfsimilarity characteristics of LTE and LTE-advanced cellular networks traffic, also they evaluated and compared selfsimilarity degree for both networks and compared user traffic with traditional voice traffic. They summarized from different data sets that the arrival pattern of the user in real LTE networks follows Poisson process. Also, they found that the inter arrival time follows the Exponential distribution. Based on our knowledge there aren't any previous studies that used sporadic and heavy tail characteristics of the LTE cellular traffic in one model. With sporadic input traffic feed to the eNodeB queue, we derived performance metrics such as mean packet delay and blocking probability analytically and verified that using the NS3 simulator under various burst values (various application) with fixed utilization. We choose the power tail distribution with various truncated tail values to represent the number of packets during a request. The reliability function of the power tail distribution used is: 


$$
R(x):=\frac{1-\theta}{1-\theta^{T}} \sum_{j=0}^{T-1} \theta^{j} \exp \frac{-\mu x}{\lambda^{j}}
$$

$\mathrm{T}=1$ refers to the exponential and large $\mathrm{T}$ for highly tail properties[23].

\section{LTE NETWORK SYSTEM MODEL}

The topology used for LTE networks is illustrated in Figure 1. It consists of a single evolved nodeB and a set of $\mathrm{N}$ wireless devices that access the LTE network. All of the wireless devices use the LTE wireless access technology for downloading data (using downlink) and to request and upload data (using uplink). We focus on downlink communication and assume that these devices use various mobile applications. Also the corresponding request traffic in the LTE downlink access network have various properties due to the different mobile applications used. The number of packets during a user request is a random variable and depends on the mobile application used, so the distribution of downlink entrance process will be different with each mobile application type. Ahmed et al in [24] compared various traffic model schemes to model the Internet data and how each model can capture behavior of the real application traffic. they showed that the bursty traffic introduced in [23] is the best distribution to model various application types due to its various parameters. The model can represent the bursty traffic (with the idle and active periods) as in Figure 2 and also produces the self-similarity property which presents many modes of use (continuous flow, Bulk arrival, Poisson arrival etc) [17, 19].

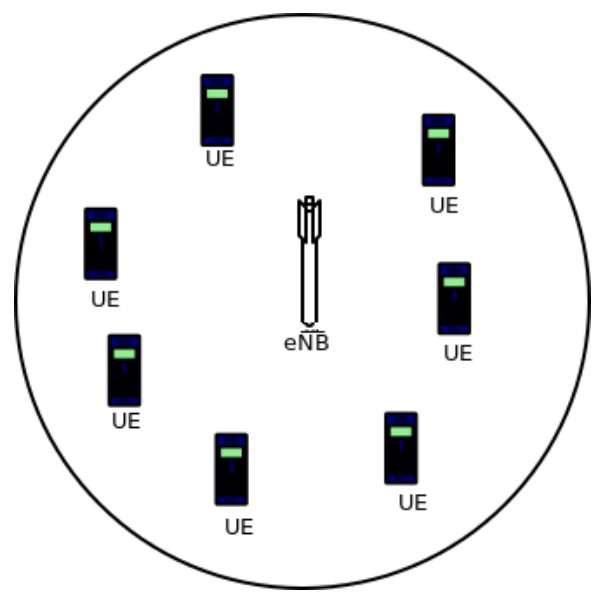

Figure 1. LTE network topology

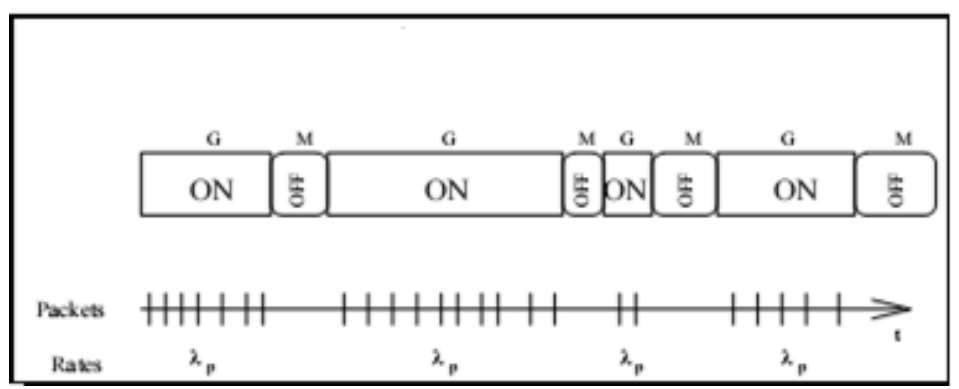

Figure 2. ON/OFF Model

The essential parameters of the traffic model used are as introduced in [24]:

$\mathrm{K}:=$ the average rate of packets arrival of connected LTE devices.

$\lambda:=$ the aggregated rates that produced by the N-LTE devices where $\lambda=\mathrm{KN}$.

$n_{p}:=$ The average number of packets produced during in the burst. 
$\lambda_{p}:=$ the maximum rate that a device can send during an active period.

$\overline{O N}:=n_{p} / \lambda_{p}=$ average time for an active period.

$\overline{O F F}:=$ average idle time between two burst sending periods.

As shown in Figure 3 the LTE network consists of the evolved packet node (eNodeB) which is responsible for allocating resources to the connected devices and the service gate way (S-GW) and packet gate way (P-GW) which connects to the eNodeB through MME unit and from MME to the internet. Also the connected devices (smart-phones) with bursty traffic model for the multi-applications. We assume that all devices are constant (so no handover to other neighbor cells). We also assume that the resources allocation in the eNodeB uses the round robin scheduling so that the resources are divided equally among the users. We use the whole single LTE cell topology as in Figure 3 with Nburst/M/1 queuing model. Now we outline the single cell LTE traffic model using sporadic traffic model:

$\mu:=$ average service rate of an eNodeB.

$\mathrm{U}:=\lambda / \mu=$ Load utilization of the access eNodeB.

Also we can control on the sporadic type with parameter "b", which can get as in [23] from the following:

$$
b=1-\frac{K}{R_{p}}=1-\frac{\lambda}{n R_{p}}
$$

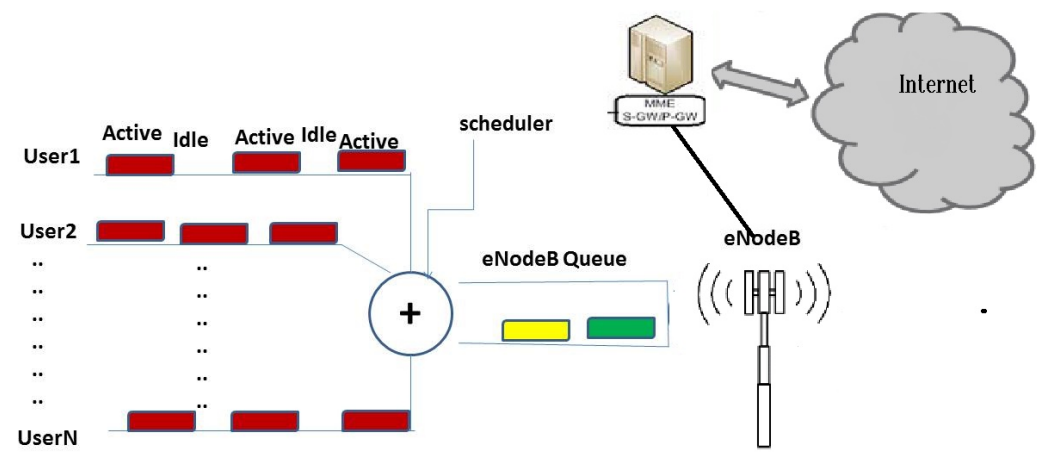

Figure 3. LTE Network Model

\section{LTE ANALYTICAL PERFORMANCE MODEL}

\subsection{Performance metrics}

The analysis of a single cell LTE access network performance with sporadic can be derived for all various distributions using matrix-exponential approach [25]. As we mentioned above that there many modes of use to our model. The first mode of use for the model where the idle period approaches zero which lead to continuous flow (no bursts and $\mathrm{b}=0)$ so the model can be reduced to Poisson arrival $\left(M_{\lambda} / M_{\mu} / 1\right.$ queueing model) and hence the delay can be derived from the following equation as in [25]: Mean Delay $(b=0)=((1 / \mu) /(1-\mathrm{U}))$ Where $\mathrm{U}=\lambda / \mu$. The second mode of use occurs when the active time approaches zero, in this case the packets arrive as a bulk arrival where "b=1". So the delay can be calculated as in [25] from equation: Mean Delay $(\mathrm{b}=1)=(\mathrm{D}(1 / \mu) /(1-\mathrm{U})), .$. where $\mathrm{D}=\frac{E\left(\frac{L(L+1)}{2}\right)}{E(L)}$. where the best performance of the cellular network is in the first mode of use where " $b=0$ ", the worst performance is in the second mode of use when " $b=1$ ". Through the analytical analysis we use fixed load utilization Ǔ at eNodeB, while we investigate the influence of bursty degree (different mobile application). The eNodeB load utilization is fixed while the size of the bursts increases or decreases according to the degree of burstiness " $b$ " to capture the influence of different applications (each value of $b$ represents a traffic type). The queueing model can be represented with matrix exponential approach of multiple application types. The steady state solution for the system can be derived as in [23] so we can get: The end to end packet delay is calculated using little's formula:

$$
\overline{D E L A Y}=\frac{1}{K} R(I-R)^{-1} \bar{\epsilon}
$$


Also we can get the block probability as follows:

$$
\text { BlockProability }=\frac{1}{K} \pi\left(R^{B} L\right) \bar{\epsilon} .(4)
$$

Where the matrix R can be calculated by solving the system as Quasi-Birth-Death Process, $\mathrm{K}$ is the average arrival rate, $\mathrm{I}$ is the identity matrix and $\epsilon$ is unity vector.

\subsection{Model analysis}

The first scenario shows the topology of the LTE network as in Figure 2. We assume that the load arrives to the eNodeB as a single flow ( $\mathrm{N}=1$ in the model in Figure 3$)$. In all scenarios we set the idle period to the exponential distribution where during active time each device produce a flow with a random size (Power tail distribution with various tail values) to represent the qualitative statistical manner of various mobile applications traffic. Setting truncated tail distribution to "1" refers to exponential distribution.

First Figure 4 shows the relation between the end to end packet delay and the burstiness parameter "b". We notice that the delay increases significantly with the increase of the burstiness parameter "b", also the delay jumps to a large value at point $(b=0.1)$ and then starts to increase gradually. More significantly the packet delay of the applications which follow distribution with tail equal to "28" is almost twice the delay of the applications that follow the exponential distribution. So, assuming that all applications will follow the exponential distribution as a service time distribution will lead to an over estimate of the LTE network capabilities. These estimates in Figure 5 are based on only a single user in the network so the effect of contention is minimum. So even with no contention, the application type has a significant impact on the packet delay of the LTE networks. Figure 5 shows the behavior of blocking probability versus the burstiness parameter "b". The figure shows clearly how the application type can affect the user block probability on the LTE networks. Clearly applications with high variability (long tails) starts to have significant blockage probability earlier than applications with small variability (exponential distributions). For small values of "b" there isn't a significant difference between various values of blockage probability for truncated tail and the exponential distribution, as burstiness parameter " $b$ " increases, the gap between the exponential and other high variability distributions increases. By taking into account (2) and (3), we can find out that the jump happen if $R_{p}>\mu$, where b $>$ "l U" at this moment.

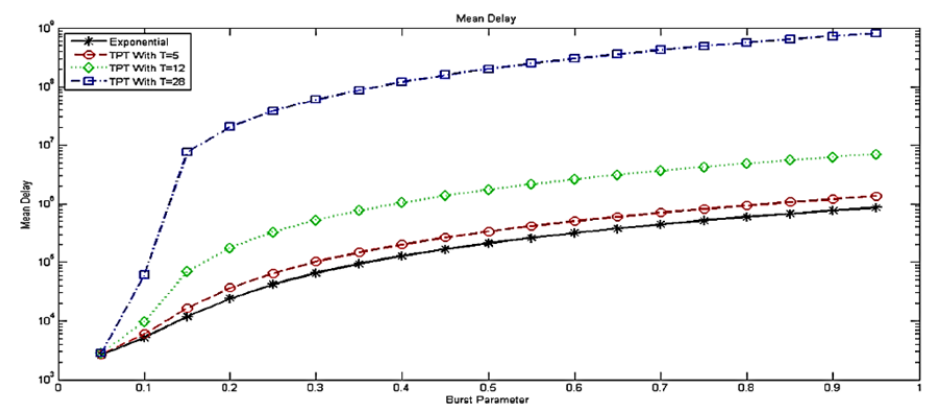

Figure 4. Single user mean delay with different burst

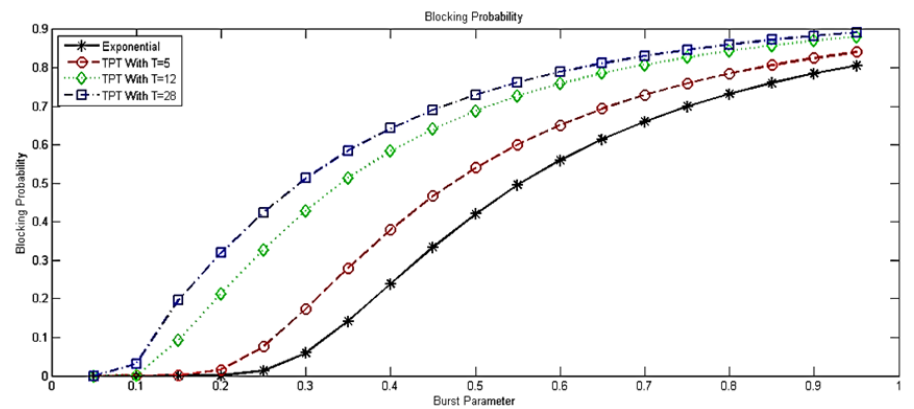

Figure 5. Single block probability 
Our next scenario as the network topology in Figure 3. We assume that the arrival at the eNodeB comes from two different sources $(\mathrm{N}=2)$, each one with $\mathrm{ON}$ periods that follow power tail distribution and exponential OFF period distribution. The integration of " $N$ " multiple exponential distributions with rate " $r$ " is $\mathrm{Nr}$ exponential distribution [25]. Hence, we can use the (N-burst/M/1) queueing model. For small values of "b", the model reduces to a continuous arrival flow with rate NK while for large values of "b" the model approaches the bulk arrival and leads to worst performance on the LTE network. To get a fair comparison with the single load source scenario, we maintain the same load utilization on the eNodeB.

The results in Figures 6 and 7 are consistent with the single flow source results. The difference in the two flow sources that users don't suffer from a large jump where the users blowup points need long bursts to occur. In case of multiple flow "N=2" we have two jump points occur as in Figure 6 for the delay and Figure 7 for the blocking probability. Hence, the performance of the delay become more complex for large values of $\mathrm{N}$. We can estimate the jump points in LTE networks with a bursty traffic source from the following equation:

$$
b=1-\frac{K}{R_{p}}=1-\frac{\lambda}{N R_{p}}
$$

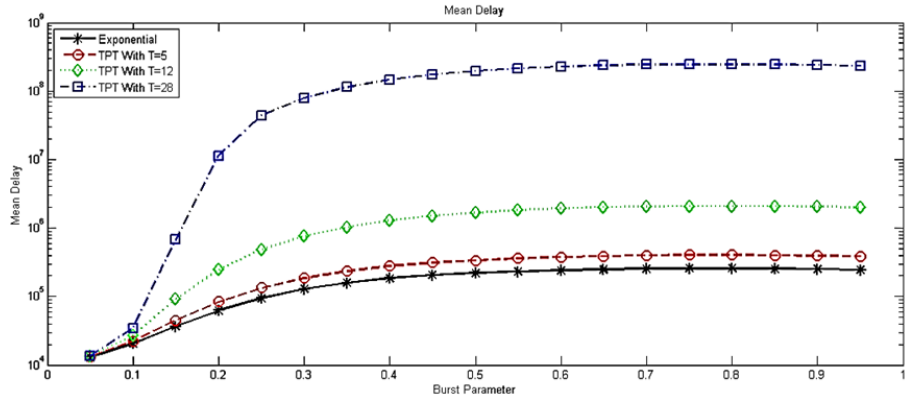

Figure 6. Multiple users mean delay with different burst

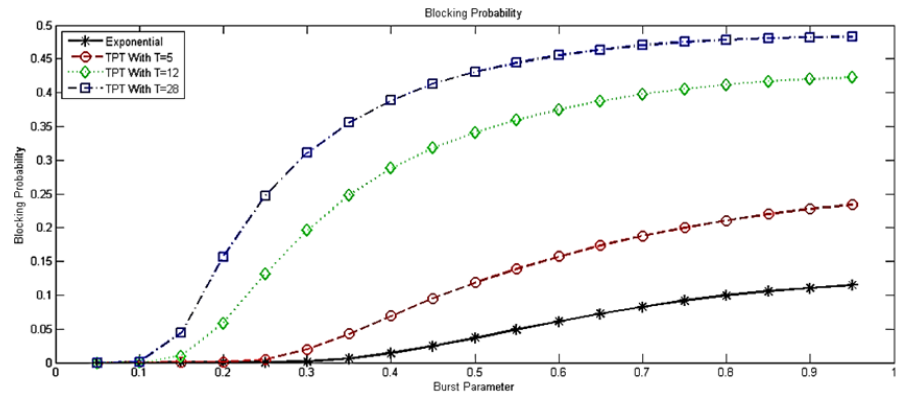

Figure 7. Multiple users block probability

\section{LTE NETWORK SIMULATION}

Our simulation consists of a single zone covered by an LTE network through one evolved NodeB (eNB) as in Figure 2 and LTE network model in Figure 3. The specification of LTE technology used in the simulator can be found in Table 1. The smartphones or subscribers are uniformly distributed in the zone according to a disc around the eNodeB. Each device generates load for amount of time (active period) and idle for another time (OFF period). We run the simulation for two scenarios with exponential OFF period distribution. The first scenario represents the normal traffic (exponential for active period) while the second scenario uses the heavy tail traffic (Pareto distribution for active period). We run the simulator for various time periods and then calculate the average over all cases.

During the active period, the number of packets in a request is a random variable with mean of 1024 byte. We refer to the number of packet with $n_{p}$ packets which are transmitted with a constant peak rate 2 
$\mathrm{Mb} / \mathrm{s}$ during an active time. Through the network simulator, we generate the different distributions from the following function:

$$
R(x)=\frac{1}{\left(1+\frac{x}{M(\alpha-1)}\right)^{x}}
$$

Where $\mathrm{R}(\mathrm{x})=\operatorname{Pr}(\mathrm{X}>\mathrm{x})$ : a reliability function of the random variable.

$\alpha: .$. a shape parameter.

$\mathrm{M}$ :..the average of the distribution, (i.e., $\mathrm{E}(\mathrm{X})=\mathrm{M})$.

\subsection{Simulation result analysis}

Figure 8 shows the aggregated throughput of the cell (eNodeB) for both the small variability distribution (exponential) and high variability (Pareto) traffic. We notice from the Figure 8 that the total aggregated throughput of LTE network for heavy traffic (Pareto) is smaller than throughput of the well behaved traffic. The result from the Figure 8 confirms with what we got in the analytical model and the cell throughput saturated at approximately" $33 \mathrm{MB} / \mathrm{s}$ " which is close to the standard throughput set in Table 1 [26]. Figure 9shows the number of users demand over time. It is very clear from Figure 9 that the large negative influence of heavy tail applications on the performance of LTE networks. The well-behaved applications network can serve more users than the network with heavy tail applications. The Figures 10 and 11 show changes of packet loss percentage per user over time. The figures clearly show that a large part of users lost $50 \%$ of their requests in Pareto distribution while the number decrease to $13 \%$ on the exponential distribution. The same consistence behavior is shown in Figures 12 and 13 for the average delay, where the delay for heavy tail distributions reach $90 \%$ of user while for well-behaved application the large delay effects $70 \%$ of users.

Table 1. LTE parameters setting

\begin{tabular}{lc}
\hline Carrier frequency.. &. $.2 .6 \mathrm{GHZ}$ \\
Bandwidth.. &. $.10 \mathrm{MHz}(50 \mathrm{RB})$ \\
Height eNB.. &. $.25 \mathrm{~m}$ \\
Transmission Power eNB & $46 \mathrm{dBm}$ \\
MIMO/SISO.. &.. $\mathrm{SISO}$ \\
eNB noise figure & $5 \mathrm{~dB}$ \\
Transmission power devices & $24 \mathrm{~dB}$ \\
UE noise figure & $7 \mathrm{~dB}$ \\
Scheduler- HARQ & Round robin Scheduler-Yes \\
Transmission model RLC & $\mathrm{UM}$ \\
Propagation Loss Model & TwoRayGround PropagationLoss Model \\
range & $150 \mathrm{~m}$ \\
Antenna model & Isotropic \\
\hline
\end{tabular}

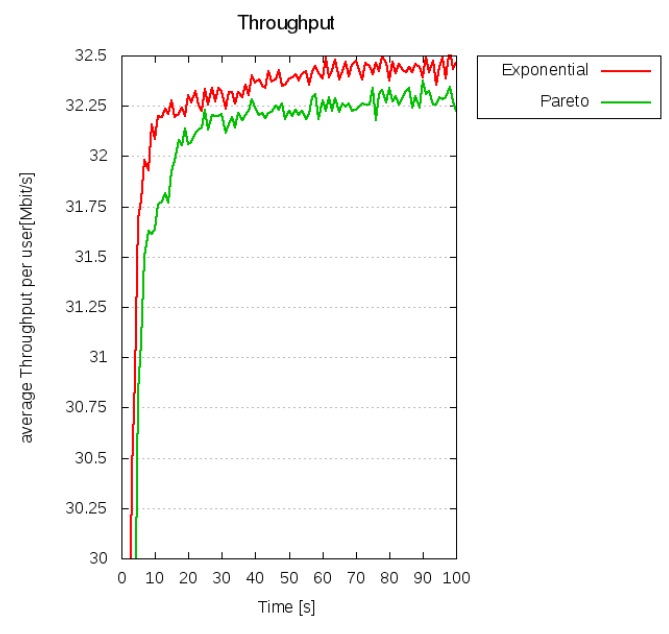

Figure 8. Throughput

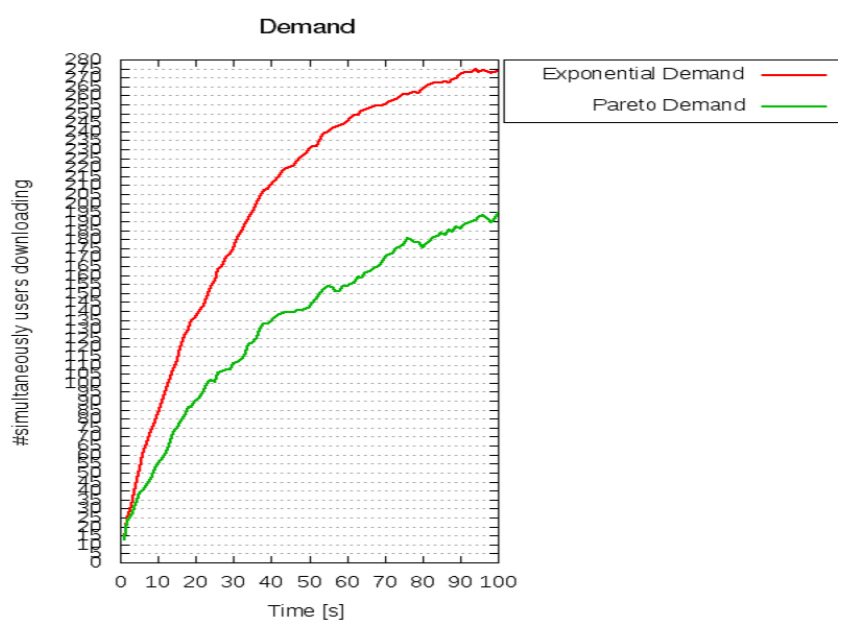

Figure 9. Demand 


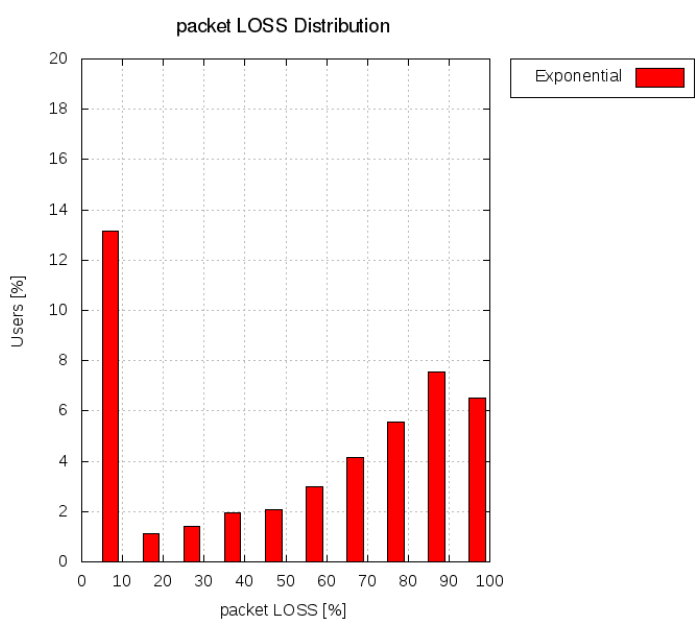

Figure 10. Packet loss distribution(EXP

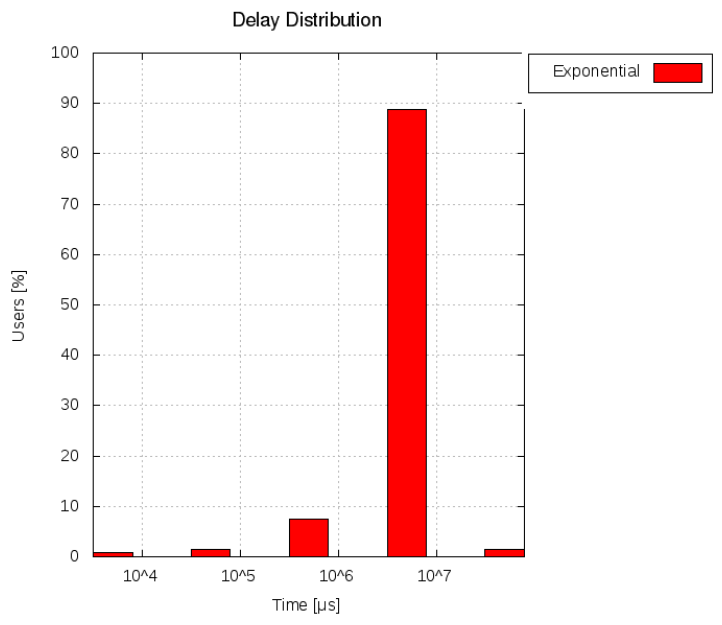

Figure 12. Delay distribution(EXP)

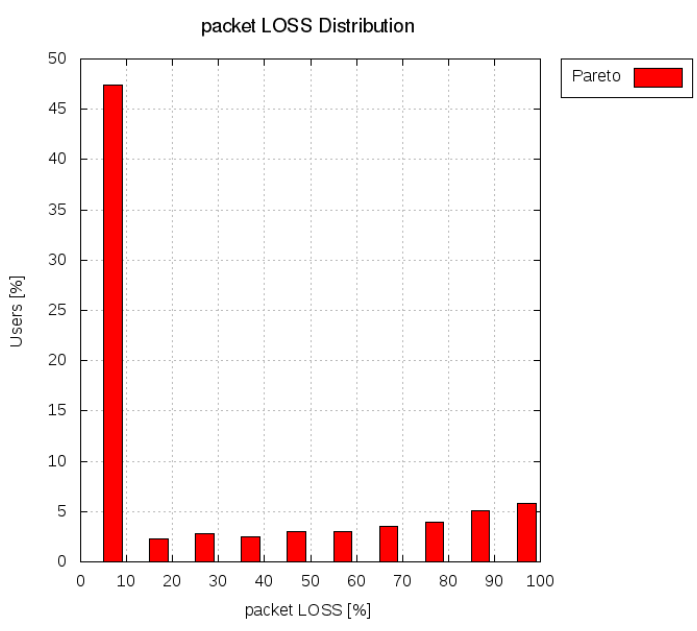

Figure 11. Packet loss distribution(Pareto)

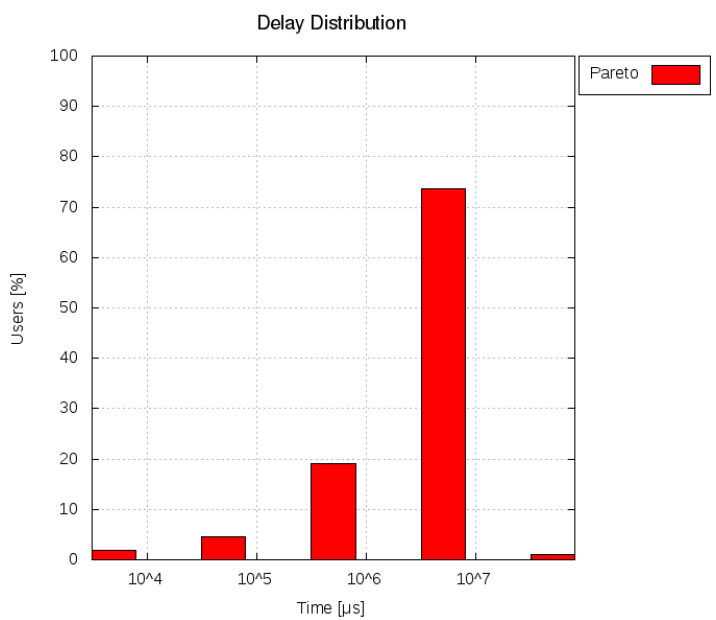

Figure 13. Delay distribution(Pareto)

\section{CONCLUSIONS}

We investigated the influence of various types of mobile application traffic on the performance of LTE mobile networks. Our research focused on the heavy-tailed and self-similar statistical characteristics of the mobile applications and its negative effect on the LTE network performance. Using the (N-Burst/M/l) Queuing model and NS3 simulator, we estimated the influence of application types on LTE cellular network performance behavior. Specifically, we studied LTE network performance metrics such as packet delay, block probability analytically and the throughput, packet delay, packet loss and user demand by the NS3 simulator. Our future work is to extend the analytical model for heterogeneous network taking into account the mobility model for wireless devices. Also to evaluate more complicated scenarios with NS3 simulator to accurately estimate actual network traffic.

\section{REFERENCES}

[1] Index, Cisco Visual Networking, "Cisco visual networking index: Global mobile data traffic forecast update 2015-2020 White Paper," Accessed date, 2016. 
[2] J. Wu, C. Yuen, N.-M. Cheung, J. Chen, and C. W. Chen, "Enabling adaptive high-frame-rate video streaming in mobile cloud gaming applications," IEEE Trans. Circuits Syst. Video Techn., vol. 25, no. 12, pp. 1988-2001, 2015.

[3] N. Panwar, S. Sharma, and A. K. Singh, "A survey on 5g: The next generation of mobile communication," Physical Communication, vol. 18, pp. 64-84, 2016.

[4] A. Gupta and R. K. Jha, "A survey of 5g network: Architecture and emerging technologies," IEEE access, vol. 3, pp. 1206-1232, 2015.

[5] M. S. Mushtaq, S. Fowler, B. Augustin, and A. Mellouk, "Qoe in 5 g cloud networks using multimedia services," Wireless Communications and Networking Conference (WCNC), pp. 1-6, 2016.

[6] I. Norros, "On the use of fractional brownian motion in the theory of connectionless networks," IEEE Journal on selected areas in communications, vol. 13, no. 6, pp. 953-962, 1995.

[7] A. Azarfar, J.-F. Frigo, and B. Sanso, "Analysis of cognitive radio networks based on a queueing model with server interruptions," IEEE International Conference on Communications, pp. 1703-1708, 2012.

[8] A. Azarfar, J.-F. Frigon, and B. Sans 'o, "Dynamic selection of priority queueing discipline in cognitive radio networks," Vehicular Technology Conference (VTC Fall), pp. 1-5, 2012.

[9] Q. Yang, J. Zheng, and L. Shen, "Modeling and performance analysis of periodic broadcast in vehicular ad hoc networks," Global Telecommunications Conference (GLOBECOM 2011), pp. 1-5, 2011.

[10] S. Yang, C. K. Yeo, and B. S. Lee, "Predictive scheduling in drive-thru networks with flow-level dynamics and deadlines," IEEE International Conference on Communications (ICC), pp. 1-5, 2011.

[11] M. J. Khabbaz, W. F. Fawaz, and C. M. Assi, "A simple free-flow traffic model for vehicular intermittently connected networks," IEEE Transactions on Intelligent Transportation Systems, vol. 13, no. 3, pp. 1312-1326, 2012.

[12] G. Comert and M. Cetin, "Analytical evaluation of the error in queue length estimation at traffic signals from probe vehicle data," IEEE Transactions on Intelligent Transportation Systems, vol. 12, no. 2, pp. 563-573, 2011.

[13] M. J. Khabbaz, W. F. Fawaz, and C. M. Assi, "Modeling and delay analysis of intermittently connected roadside communication networks," IEEE Transactions on Vehicular Technology, vol. 61, no. 6, pp. 2698-2706, 2012.

[14] M. K. Karray and M. Jovanovic, "A queueing theoretic approach to the dimensioning of wireless cellular networks serving variable-bit-rate calls," IEEE Transactions on Vehicular Technology, vol. 62, no. 6, pp. 2713-2723, 2013.

[15] H. Yang and B. Sikdar, "Queueing analysis of polling based wireless mac protocols with sleep-wake cycles,” IEEE Transactions on Communications, vol. 60, no. 9, pp. 2427-2433, 2012.

[16] B. Shrader and A. Ephremides, "Queueing delay analysis for multicast with random linear coding," IEEE Transactions on Information Theory, vol. 58, no. 1, pp. 421-429, 2012.

[17] Y.-C. Chen, J. Kurose, and D. Towsley, "A mixed queueing network model of mobility in a campus wireless network,” Proceedings IEEE INFOCOM, pp. 2656-2660, 2012.

[18] S. Fowler, C. H. Hall, D. Yuan, G. Baravdish, and A. Mellouk, "Analysis of vehicular wireless channel communication via queueing theory model," IEEE International Conference on Communications (ICC), pp. 1736-1741, 2014.

[19] S. Fowler, J. Sarfraz, M. M. Abbas, and V. Angelakis, "Gaussian semi-markov model based on real video multimedia traffic,” IEEE International Conference on Communications (ICC), pp. 6971-6976, 2015.

[20] N. N. Sirhan, M. Martınez-Ramon, G. L. Heileman, N. Ghani, and C. C. Lamb, "Qos performance evaluation of disjoint queue scheduler for video-applications over lte-a hetnets," Proceedings of the 7th International Conference on Computing Communication and Networking Technologies, pp. 4, 2016.

[21] V. Naumov, K. Samouylov, N. Yarkina, E. Sopin, S. Andreev, and A. Samuylov, "Lte performance analysis using queuing systems with finite resources and random requirements," 7th International Congress on Ultra Modern Telecommunications and Control Systems and Workshops (ICUMT), pp. 100-103, 2015.

[22] R. K. Polaganga and Q. Liang, "Self-similarity and modeling of lte/lte-a data traffic," Measurement, vol. 75, pp. 218-229, 2015.

[23] H.-P. Schwefel and L. Lipsky, "Performance results for analytic models of traffic in telecommunication systems, based on multiple ON-OFF sources with self-similar behavior," Teletraffic science and engineering, pp. 55-65, 1999. 
[24] Ahmed M. Mohamed and , Adel F. Agamy, "A survey on the common network traffic sources models," Interenational Journal Of Computer Network (IJCN), vol. 3, no. 2, 2011.

[25] L. Lipsky, "Queueing Theory: A linear algebraic approach," Springer Science Business Media second Edition, 2008.

[26] E. Dahlman, S. Parkvall, and J. Skold, "4G: LTE/LTE-advanced for mobile broadband," Academic press, 2013.

\section{BIOGRAPHIES OF AUTHORS}

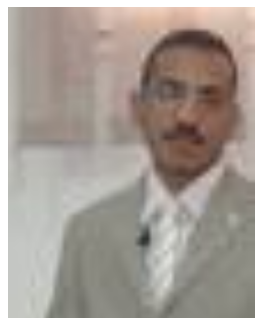

Adel Agamy received the B.Sc. in Communications and Electronics engineering in 2007, M.Sc. in Electrical Engineering in 2012 from Aswan university, Egypt. He is currently an assistant lecturer at electrical engineering department, Aswan faculty of engineering, Aswan University. His fields of interest: traffic modeling, wireless communication, cellular network and computer network

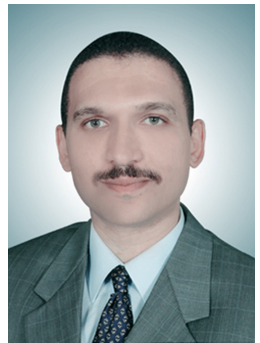

Ahmed Mohamed received the B.Sc. in electrical and computer engineering from Assiut university, Egypt, in 1994, the M.Sc. in computer science and engineering from university of Connecticut, USA in 2001 and the Ph.D. in computer science and engineering from university of Connecticut, USA in 2004. He is currently an assistant professor at electrical engineering department, Aswan faculty of engineering, Aswan University. His research interests include, performance modeling, queueing analysis, distributed systems, computer networks and operating systems. 